\title{
L'avenir de la médecine appartient aux soins intégrés
}

Contrairement à l'impression que donnent les nombreux courriers des lecteurs, plus de la moitié des médecins de famille travaillent déjà aujourd'hui selon des modèles Managed Care et la tendance est à la hausse. Les soins intégrés renforcent les soins de base et offrent un potentiel d'amélioration de la situation économique des médecins de famille. Les patients et les assureurs en profitent également.

Heinrich Zürcher

Membre du Directoire d'Argomed Ärzte AG
Correspondance: Dr Heinrich Zürcher Argomed Ärzte AG Täferstrasse 16 CH-5405 Dättwi

heinrich.zuercher@hin.ch
De nombreux courriers de lecteurs publiés dans le Bulletin des médecins suisses reflètent une aversion pour Managed Care. Ces opinions négatives masquent le fait que Managed Care revêt dès à présent une grande importance pour les médecins de famille. Plus de la moitié d'entre eux s'engagent volontairement dans des modèles Managed Care. Près de 20\% des assurés ont déjà pris confiance dans ces modèles d'assurance. Depuis sa création il y a 12 ans, Argomed s'engage en faveur de Managed Care. Nos expériences sont globalement positives et à notre avis les aversions résultent majoritairement de préjugés et d'un déficit d'information.

\section{Facultatif et indépendant}

Managed Care est basé sur le volontariat pour les médecins comme pour les assurés et cela ne doit pas changer. Les patients peuvent choisir leur médecin, de quelques rares HMO appartenant aux caisses, tous les réseaux de médecins sont autonomes et ne reçoivent pas d'ordre des assureurs-maladie.

\section{Managed Care est synonyme de médecine de qualité}

Quiconque participe à un réseau de médecins s'engage à participer à un cercle de qualité. Ce qui peut ressembler à une obligation pesante s'avère être une activité amusante. Les thèmes médicaux que le cercle de qualité se fixe lui-même sont prioritaires. L'échange de réflexions («comment faites-vous?») débouche sur un consensus, voire sur un guide. Les sujets d'ordre économique sont d'importance secondaire. L'échange d'expériences revêt une grande importance. L'évolution des soins de santé régionaux jusqu'à la politique de santé cantonale et fédérale est évoquée. Les spécialistes ont la possibilité de s'exprimer et de contribuer

\section{L'échange de données entre les partenaires doit être organisé de façon professionnelle pour que la charge administrative reste modérée pour nous en tant que médecins.}

même dans les modèles Managed Care. Ils conservent leur médecin de famille personnel et peuvent parfaitement accéder aux prestations utiles. A quelques rares exceptions près, ils s'engagent à consulter toujours en premier leur médecin de famille (Gatekeeping). Les patients qui ne souhaitent pas procéder ainsi ont toujours la possibilité de payer autour de 500 à 700 francs de plus par an pour une consommation médicale non surveillée. La participation est également facultative pour les médecins de famille. Quiconque n'est pas convaincu des mérites de Managed Care peut s'en tenir à l'écart. Ceux qui optent pour une participation ont le choix entre les modèles HMO et le médecin de famille. A l'exception aux critères d'affectàtion dans le cadre du réseau de médecins. Nous développons notre propre opinion concernant les thèmes de la politique de la santé et de la profession. Les médecins bien organisés sont des médecins bien informés!

\section{Les médecins bien organisés se font entendre} Aussi démocratique qu'elle soit, la réflexion sur les courriers de lecteurs publiés dans le Bulletin des médecins suisses se limite à des avis personnels. Cette controverse n'est pas adaptée pour influer sur la politique de santé. Pour cela, nous avons besoin d'organisations dynamiques disposant de ressources professionnelles. C'est le seul moyen pour faire jeu égal avec 
les organismes d'Etat, les assureurs et les hôpitaux. Managed Care a permis la constitution de réseaux de médecins régionaux qui se sont regroupés au sein de sociétés d'exploitation. Les organisations professionnelles des médecins de premier recours s'en sont trouvées renforcées. Grâce à Managed Care, nous disposons aujourd'hui d'organisations qui défendent les intérêts spécifiques des médecins de famille (et d'ailleurs même des médecins non Managed Care).

\section{Les soins intégrés sont le reflet d'une méde- cine moderne}

La collaboration entre médecins est empreinte de valeurs bien établies telles que la collégialité, l'estime et le respect. Ces principes ne devraient pas souffrir de la pression économique. Ils permettront également à l'avenir de convenir d'un commun accord d'une collaboration interdisciplinaire. Chaque acteur accomplit le travail qui relève de ses compétences. La parfaite collaboration est un impératif de notre époque et s'avère vitale pour nos patients. La collaboration informelle traditionnelle fait que de nombreux patients glissent à travers les mailles du filet. La collaboration évolue à présent en direction d'un réseau de soins structuré, sûr et bien organisé avec des contrats de collaboration qui seront également indispensables pour les futurs DRG.

\section{Le rôle des organisations Managed Care des médecins}

Des contrats entre les assureurs et les réseaux de médecins constituent la condition sine qua non des soins intégrés. De tels contrats ne peuvent plus être négociés par les comités des associations dans le cadre d'un système de milice. L'échange de données entre les partenaires doit également être organisé de façon professionnelle pour que la charge administrative reste modérée pour nous en tant que médecins. Il s'agit de missions qui incombent aux organisations Managed Care des médecins, par exemple aux sociétés d'exploitation. Des prestations complémentaires au-delà des standards TARMED peuvent également être proposées et rémunérées en dehors du tarif. A l'avenir, les médecins de famille assureront une partie de leurs revenus par le biais du rôle qu'ils joueront dans la médecine intégrée.

\section{Des signaux positifs pour la relève}

Trop longtemps nous nous sommes lamentés de notre mauvaise situation. Au moins, le public en a-t-il pris conscience, même si la situation économique n'a pas encore évolué d'une façon satisfaisante. Nous

\begin{abstract}
Argomed
Argomed est la plus grande organisation médicale de Managed Care (MC) et compte 600 médecins répartis en 18 réseaux de médecins dans plusieurs cantons avec 200000 assurés MC. L'offre de prestations diversifiées est issue de I'assistance à la négociation MC et de l'administration MC avec analyse des données MC. Les cercles de qualité et les réseaux de médecins bénéficient d'un soutien logistique. Des offres complémentaires innovantes allant au-delà de TARMED sont mises au point suivies d'une indemnisation correspondante.

«MehrFachArzt» représente le seul label de qualité développé uniquement pour les médecins de famille et constitue une nouvelle offre. «MehrFachArzt» est le certificat afférent aux critères de qualité vraiment importants des médecins de famille. La promotion de la cybersanté avec le dossier médical électronique et l'échange de données électronique constitue une autre mesure de soutien des médecins de famille. Des contrats de collaboration sont conclus avec les hôpitaux et les groupes de médecins spécialisés. CIRS est le système d'annonce des événements critiques. Le projet KIMSA visant à annoncer les processus de guérison potentiellement longs a été mis au point avec la SUVA. Argomed propose désormais aussi une aide pour la mise en place et l'exploitation de cabinets de groupe.
\end{abstract}

avons en revanche découragé la relève. Une certaine lueur d'espoir se dessine à l'horizon (cabinets de groupe, activité à temps partiel, rémunération des activités dans le cadre des soins intégrés). Les soins intégrés constituent une opportunité pour améliorer notre situation économique.

\section{Conclusion}

Aujourd'hui, déjà plus de la moitié des médecins de premier recours participent volontairement à Managed Care. La participation permet d'adhérer à un groupe de médecins engagés dans l'intérêt direct de chaque médecin de famille mais aussi de ses patients. Le mouvement des médecins de famille est fort et progresse. Des structures professionnelles assurent son dynamisme et renforcent son influence sur le système de santé. Ce climat positif aura pour conséquence de lever les derniers a priori contre Managed Care. 